\title{
Non-additive components of merit in crossbred mice
}

\author{
B. KINGHORN \\ Institute of Animal Breeding and Genetics, Agricultural University of Norway \\ Box 24, $1432 \stackrel{\circ}{A} s-N L H$, Norway
}

Three inbred strains of mice were crossbred following many mating systems, generating 65 genotypes $(\times 2$ sexes) by the third generation when measurements of fertility, mortality, body weight and tail length were taken. These data were analysed by five models involving additive and dominance effects (model 1), additive, dominance and epistatic effects (models 2, 3 and 4), and genotype classification (model 5). Inclusion of epistatic effects in models generally made efficient use of the extra degrees of freedom used up. Data from future generations is required to estimate epistatic parameters with reasonable accuracy.

\section{The use of non-additive components in selection policy}

\author{
B. KINGHORN and H. SKJERVOLD \\ Institute of Animal Breeding and Genetics, Agricultural University of Norway \\ Box 24, 1432 As-NLH, Norway
}

This paper discusses the use of knowledge about breed composition of individuals when carrying out selection in a synthetic population. Formulae are given for the optimum overall breed composition of the synthetic and for the approximate number of breeds which should be involved. The extra genetic variation created in a synthetic population is presented in two components - one due to breed composition of individuals and the other due to breed composition at each locus involved. The basis of a selection index procedure incorporating additive and non-additive (due to breed composition) components is proposed.

\section{Problems of interpreting heterosis from results of wide crosses in sheep}

\author{
G. NITTER \\ Institut für Tierhaltung und Tierzü̈chtung, Univ. Hohenheim \\ D-7000 Stuttgart 70
}

Some reproductive traits were compared in three female sheep groups where one was German Merinolandschaf and the two others $F_{1}$ and backcross generations to the genetically widely distinct Finnish Landrace breed. The possible bias of parameter estimation (breed difference, heterosis) due to recombination loss and detrimental maternal effects in the backcross generation were discussed. Furthermore, the predictive value of the parameters was questioned in view of an obvious environmental insufficiency for one of the test groups. 